\title{
Optimization of a native gel electrophoretic process for the purification of intracellular green fluorescent protein from intact Escherichia coli cells
}

\begin{abstract}
Intracellular green fluorescent protein (GFP) can be separated and purified from intact Escherichia coli cells by a preparative native polyacrylamide gel electrophoresis (n-PAGE). The effects of operating parameters such as the volume and concentration of feedstock and the pore size and height of resolving gel on the purity and yield of GFP were studied using a $1.7 \mathrm{~cm}$ internal diameter gel column. The optimum conditions for this preparative n-PAGE operation were determined to be $100 \mathrm{\varepsilon l}$ of $15 \%$ (w/v) feedstock and a $12 \%(\mathrm{w} / \mathrm{v})$ resolving gel with a gel height of $2 \mathrm{~cm}$. The purity and yield of the recovered GFP were 98 and $88 \%$, respectively. The results of scalability studies show that the ratio of feedstock volume to cross-sectional area of the column is an important consideration for scaling up the preparative n-PAGE.
\end{abstract}

Keyword: Escherichia coli; Green fluorescent protein; Optimization; Preparative native polyacrylamide gel electrophoresis; Protein purification; Scale-up criterion 